\title{
Assessing the use of social media as a source of information related to dentistry in Saudi Arabia
}

\begin{abstract}
The term social media usually refers to Internet tool that allows communication between individuals, and enables them to share information, opinion, photos, videos, and other content.$^{1}$ Social media, offering a public reservoir of information, may serve as a source of patient information to aid clinical care. ${ }^{2}$ Patients are increasingly active online, and they are frequent social media users for health purposes. In Saudi Arabia, social media and technology is increasingly popular, mostly due to the low average age of the country's population. ${ }^{3}$ The aim of this study is to assess the use of social media of Saudi population, and to assess which social media platform are used more often. In addition to the use of social media as source of information that are related to dentistry in Saudi Arabia with the associated factors.
\end{abstract}

Material and method: The study used an Internet-based Arabic language questionnaire, with participants self-recruited by snowball sampling via social media networking sites. Our study was aimed at all residence in Saudi Arabia above 18 years of age who could be accessed through social networking sites.

Results: A total of 1030 responses were received, where females constituted $80.5 \%(860)$ of the total respondent. The age range of the majority of the survey respondent's was from $29-39$ years old $(39.9 \%$ ), followed by $34.3 \%$ at the age range of $18-28$ years, $17.2 \%$ were at the age range of $40-50$ years and the least were above 50 years of age represented with $8.6 \%$. Around ninety eight percent of the respondents reported that they are using or browsing social media websites and $92.2 \%$ reported that they have personal accounts on the social media. About fifty eight percent of the respondents reported that they search on social media if they faced any dental issue, and $68.6 \%$ of them stated that they usually find the information they were looking for.

Conclusion: Social media is bringing a new dimension to the health care area by offering a medium to be used by patients, and health care professionals. These networks are bringing health information to many more people than ever before. And they are widening access to those who may not easily access health information via traditional methods, such as younger people, minorities, and lower socioeconomic groups.
Volume 8 Issue 7 - 2017

\author{
Mayyadah Almozainy \\ Department of Restorative Dentistry, King Saud University, \\ Saudi Arabia
}

Correspondence: Mayyadah Almozainy, Department of Restorative Dentistry, King Saud University, P.O.Box 2456, Riyadh I I45I, Saudi Arabia, Email mmozainy@yahoo.com Received: November 28, 2017 | Published: December 07,
2017

\section{Introduction}

The term social media usually refers to Internet tool that allows communication between individuals, and enables them to share information, opinion, photos, videos, and other content. ${ }^{1}$ There are various kinds of social media platforms such as blogs like Tumbler and Blogger, social networks similar to Facebook, Twitter, MySpace and Google, professional networks such as LinkedIn, media sharing as YouTube and Flickr, collaborative projects like Wikipedia, and virtual gaming or social worlds as Second Life and Human Sim..$^{4-6}$ The collaborative environment to which social media belongs represents a shift in technology and functionality from 'Web 1.0', in which static online content and applications were created and published by individuals, to 'Web 2.0', in which there is continuous modification and participation by all users. ${ }^{7}$ Different social media attract different age groups, for example, blogging has become less popular among teens and young adults since 2006 and more popular within older adults. ${ }^{89}$ Similarly, there are differences in social media use depending on gender, race/ethnicity, education, and income characteristics. ${ }^{10}$ Social media, offering a public reservoir of information, may serve as a source of patient information to aid clinical care. ${ }^{2}$ It is clear, though, that in addition to seeking out traditional sources of healthcare information, patients are increasingly active online. ${ }^{11}$ In 2011, looking for healthcare information was the third most common online activity, ${ }^{12}$ and in September 2012, 72\% of adult internet users sought support and medical information online. ${ }^{13}$ In $2012,67 \%$ of internet users were using social media for any purpose ${ }^{14}$ and $26 \%$ were using it for health issues. ${ }^{13}$ By transcending geographical boundaries, social media has facilitated extension of clinical care to distant patients. ${ }^{2}$ Telemedicine, broadly defined as using telecommunication for patient care, has been around since the 1960s but has only recently experienced rapid growth thanks to new technologies. ${ }^{15}$ Patients are increasingly active online, and they are frequent social media users for health purposes. In 2015 , it has been estimated that $62 \%$ of the entire adult population of the USA uses Internet and $72 \%$ of adult Internet users use Facebook; ${ }^{16}$ a previous survey by Pew Internet \& American Life Project reported that $72 \%$ of U.S. adults looked online for health information. ${ }^{17}$ Physicians are also using Facebook pages, Twitter accounts, and blogs in hopes to grow their practice and earn patient referrals. ${ }^{18}$ In addition, patients can now rate their physicians on any of a multitude of physician rating sites, enabling patients to enter reviews and ratings of their physicians, freely available for anyone to see. Most sites provide some basic information about the physician, such as years in practice and contact information, with some 
also including training. In a US study, $37 \%$ had consulted physician rating sites when gathering information about a specific provider. ${ }^{19}$ Advocates of the use of social media in healthcare suggest that these tools allow for personalization, presentation and participationthree key elements that make them highly effective. ${ }^{19,20}$ The content can be tailored to the priorities of the users; the versatility of the different platforms creates numerous options for the presentation of information, and the collaborative nature of social media allows for a meaningful contribution from all user groups. The idea of a synergistic relationship between social media users is one of the main perceived advantages of using these platforms. ${ }^{21}$ However, criticisms of the use of social media in healthcare have also arisen. The availability of misinformation is a risk, as healthcare providers are unable to control the content that is posted or discussed.22-24 Inappropriate substitution of online information or advice for in-person visits to a healthcare provider can also potentially lead to harmful results, and this has been cited as a limitation of the use of social media and of the internet generally. ${ }^{22-25}$ Negative uses of social media have also been highlighted in the context of professionalism and confidentiality, ${ }^{26} \mathrm{In}$ Saudi Arabia, social media and technology is increasingly popular, mostly due to the low average age of the country's population. ${ }^{3}$ The aim of this study is to assess the use of social media of Saudi population, and to assess which social media platform are used more often. In addition to the use of social media as source of information that are related to dentistry in Saudi Arabia with the associated factors.

\section{Materials and methods}

The study used an Internet-based Arabic language questionnaire, with participants self-recruited by snowball sampling via social media networking sites. Our study was aimed at all residence in Saudi Arabia above 18 years of age who could be accessed through social networking sites. A draft questionnaire was constructed in English and translated into the native language (Arabic). Face validity was established as both English and Arabic versions of the questionnaire were checked several times to evaluate whether the questions effectively capture the topic under investigation by an expert, in addition the survey was checked for common errors like double-barreled, confusing, and leading questions by a psychmetrician. Then a pilot testing was done on 60 participants, accordingly, the questionnaire was uploaded into a professional online survey (Google forms). The link to complete the questionnaire online was made available through the most popular social networking sites in Saudi Arabia: Twitter, Snap chat, Instagram and Facebook. Information about the survey and the confidentiality of responses was made clear in the covering letter. All the participants were requested to forward the link to their friends and families to recruit as many participants as possible. The responses collected were retrieved from the online survey software for data analysis after the closing date of the survey (November 2017). The received data was analyzed by employing descriptive statistics, including response frequencies and \%ages.

\section{Results}

A total of 1030 response was received, where females constituted $80.5 \%(860)$ of the total respondent. The majority of the survey respondent's ages ranged from 29-39 years old (39.9\%), followed by $34.3 \%$ at the age range of $18-28$ years, $17.2 \%$ were at the age range of $40-50$ years and the least were above 50 years of age represented with $8.6 \%$. About Sixty four percent of the respondents were bachelor degree holder, followed by $19.7 \%$ with high school degree or less, $15.3 \%$ are masters or PHD holders and less than one percent did not obtained any level of education. The occupational status of the participants is presented on Table 1. Monthly income rate was divided into groups starting from less than two thousand Saudi riyals and up to more than fifteen thousand Saudi riyals as presented in Figure 1. Most of the participants are living in the main cities of Saudi Arabia (95.1\%), with a majority of cluster in the central region $(65.6 \%)$, followed by western region $(14.9 \%)$, southern region $(9.3 \%),(6.2 \%)$ of the participants were living in the eastern region and the least number of respondent were living in the northern region (4.1\%). Around ninety eight percent of the respondents reported that they are using or browsing social media websites and $92.2 \%$ of them have personal accounts on the social media. The most frequently used device for social media browsing was mobile phone and $79 \%$ of the users reported having internet access within their devices. When the participants were asked about which social media applications they are usually browsing, they were given the chance to choose more than one option and the results are shown in Figure 2. Around seventy percent of the respondents reported that they are interested in obtaining information related to dentistry through social media. $31.9 \%$ reported that they did choose a dentist to visit through social media; in addition $70.2 \%$ reported that they were satisfied after their visit in comparison to what they see on the dentist's social media account. To explore more in which factors usually has higher impact on dentists' social media accounts, several factors were included in the survey and participants were able to choose more than factor as presented in Figure 3. About fifty eight percent of the respondents reported that they search on social media if they faced any dental issue, and $68.6 \%$ of them stated that they usually find the information they were looking for. The rate of participants' average use of social media applications to gain information related to dentistry is presented in Figure 4, and the number of dental social media accounts they are following is presented in Figure 5. Deferent social media applications were listed in the survey, and participants were asked which of them in their personal opinion has/have the highest impact on his/her dental knowledge, more than one option could be selected in this section as presented in Figure 6. Around seventy two percent of the respondents believe that social media is an effective tool to gain information related to dentistry.

Table I Occupational status of participants

\begin{tabular}{llll}
\hline Occupational Status & \multicolumn{4}{l}{ Male Female Total } \\
\hline Employee & 120 & 367 & $487(46.8 \%)$ \\
Student & 35 & 200 & $235(22.8 \%)$ \\
No occupation & 16 & 299 & $315(30.4 \%)$ \\
\hline
\end{tabular}

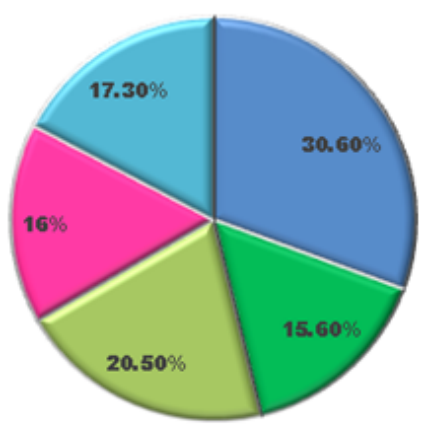

Whs than $2000 \quad M 2000-5000 \quad M 5000-1000 \quad M 10000-15000 \quad$ M more than 15000

Figure I Monthly income rate in SAR. 


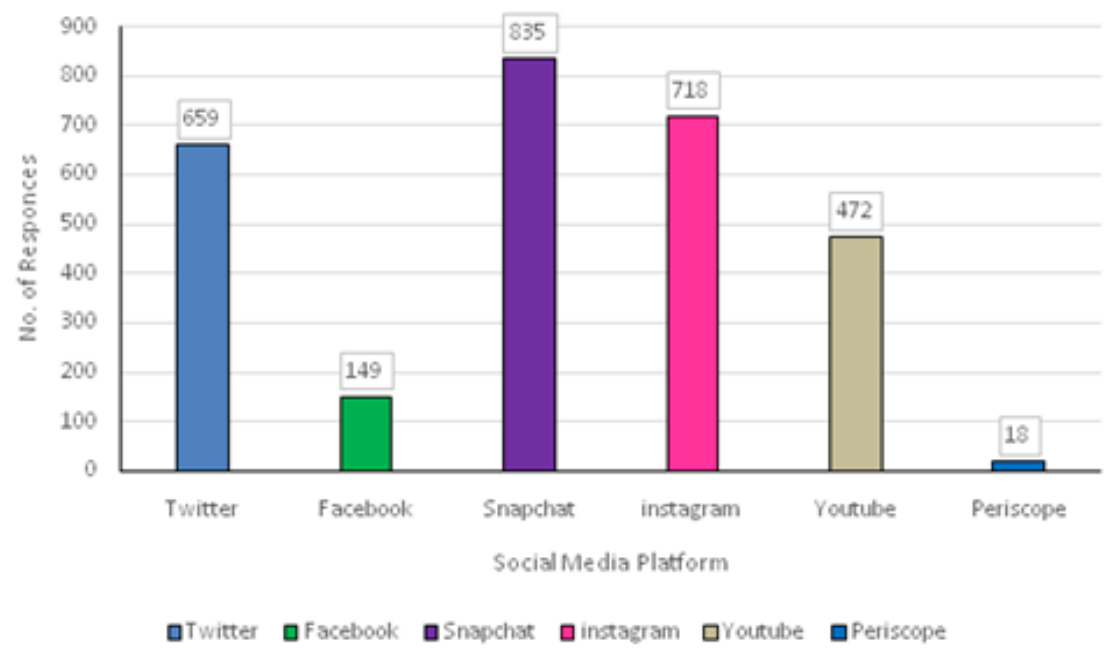

Figure 2 Social media applications used.

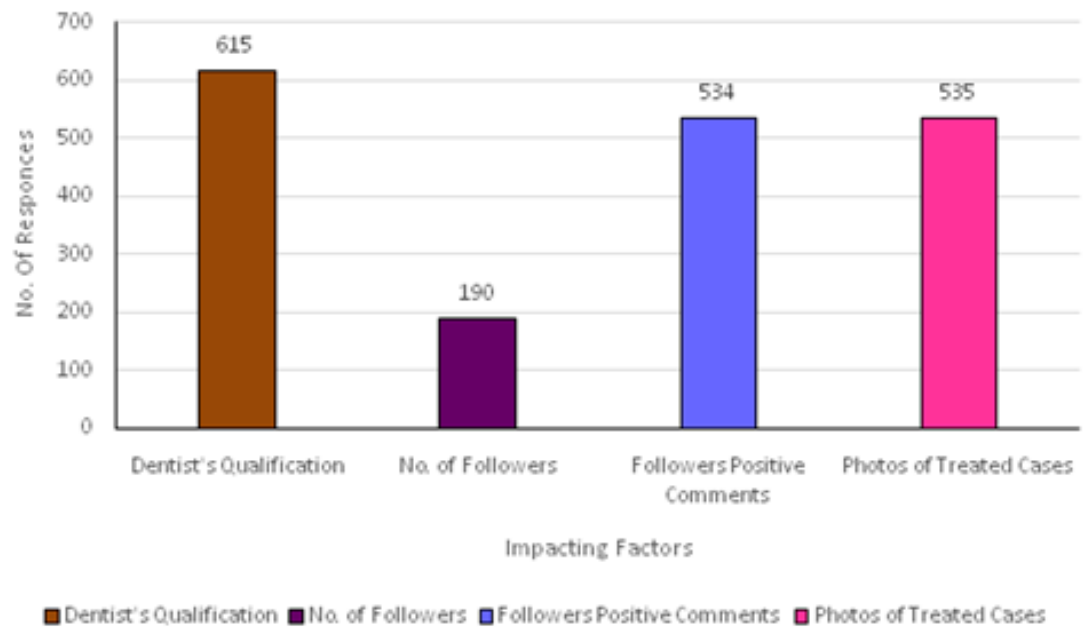

Figure 3 Impacting factors on dentists' social media accounts.

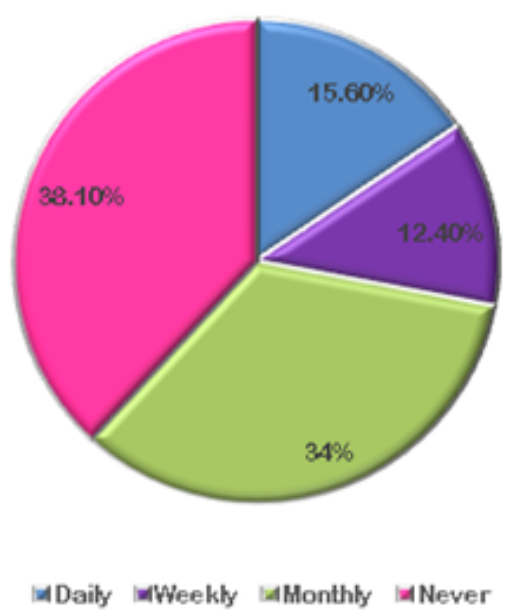

Figure 4 Average social media used to gain dental information. 


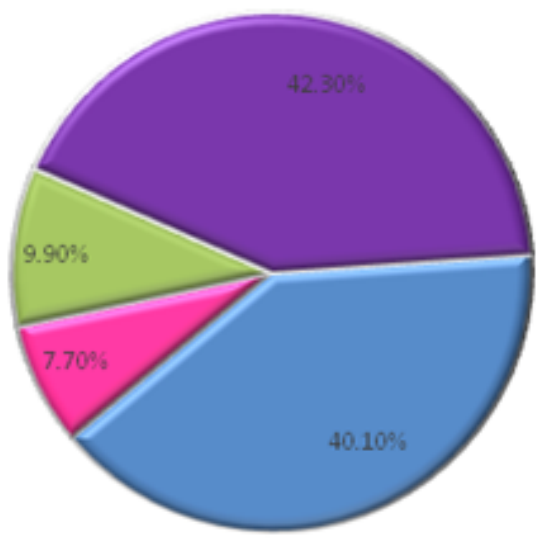

I1_5 M6_10 MMorethan 10 in Wone

Figure 5 Number of dental social media accounts followed.

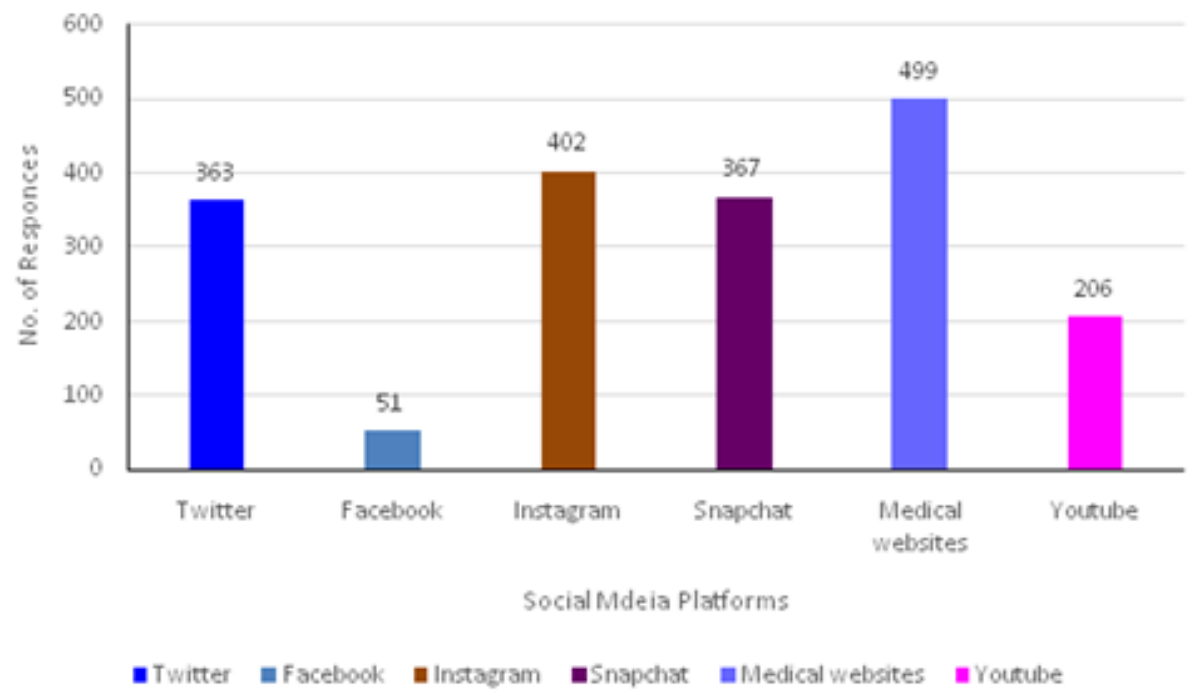

Figure 6 Social media applications impact on dental knowledge.

\section{Discussion}

As of January 2017, approximately half the world population has access to the internet, while the number of worldwide social media users is expected to exceed 2 billion in 2016. ${ }^{27}$ Although North America is the leading region in terms of internet penetration, the Middle East is also increasingly connected, with an internet penetration above the world average $-60 \%$ of the total population. ${ }^{28}$ This increase in access to internet services is largely supported by increasing access to mobile devices and decreasing costs of internet packages. In Saudi Arabia, social media and technology is increasingly popular, mostly due to the low average age of the country's population. ${ }^{3}$ The internet penetration of Saudi population have been reported to be $64 \%$ with more than ten million active social media accounts $(35 \%)$ in $2016 .{ }^{29}$ These numbers were clearly represented in the current study, where $98.8 \%$ of the respondents reported that they are using or browsing social media platforms and $92.2 \%$ of them reported that they have personal accounts on social media. Seventy nine percent of the respondents have access to the internet on their mobile phones, and mobile phones were the most frequently used device to access social media platforms. In regards to the social media platforms mostly used in Saudi Arabia, Snapchat was almost non-existent in the statistics in 2015, but it has made a major grow in 2016 where it grabs $13 \%$ of the social media users with around 4.1million active users. ${ }^{29}$ And it has been reported as the most frequently used application in our study, followed by Instagram, Twitter, YouTube and Facebook respectively. About seventy percent of the respondents reported that they are interested in receiving information related to dentistry through social media and this interest rate was almost constant between different regions of Saudi Arabia, female respondents were more interested where $72.1 \%$ of the total respondents answered with "yes" in comparison to $65 \%$ of male respondents. When age was the comparison factor, younger age group (18-28 years) was the most interested group with $75 \%$. Rate of interest has an inverse relationship with age groups where it reaches $63 \%$ at the age range of 40 to 50 years, and $65 \%$ with the oldest age group (more than 50 years). The high interest rate repotted with the youngest age groups could be a reflection of their high interest and involvement in new technologies and usually they 
are more active in social media. The monthly income rate has no impact on the participants' interest of receiving information related to dentistry through social media, since the interest reported from all the groups was higher than $60 \%$. Although the highest interest was associated with the lowest monthly income group (75.6\%). Although I was not expecting the high interest rate with the lowest monthly income group due to the fact that subscription fees for internet bundles in Saudi Arabia is relatively high in comparison to other countries, one of the reasons that could explain this result is that because they might browse the internet more often searching for information and recommendations about preventive methods or looking for the least expensive treatment modalities that could be suitable for them before they visit a dental office for consultation to save the consultation fees which is considerably high in the private sector if they does not have accessibility to governmental dental clinics. Thirty two percent of the total respondents ( $86.5 \%$ of them were females) had actually decided to visit a specific dentist based on social media, after their visit $70.2 \%$ reported that they were satisfied with their experience in comparison to what the dentist posts on social media. This high impact on females' decision rather than males reflect the high aesthetic demand usually females have. Forty eight percent of the respondents living in rural areas did choose their dentists through social media in comparison to $31 \%$ who live in major cities. That could be contributed to many factors, such as that in major cities people might choose their dentist based on friends or community recommendations, referrals, location, or have a specialized dental office that they visited and trusted in their area. In contrast to these factors, rural areas usually does not have specialized dental offices. People from these areas usually arrange their dental visits in the city, so they most probably search the web to find the best dentist for their case and make their decision based on that The results where reassuring when the participants were asked about their personal opinion in which factors have the major effect on dental social media accounts, since the number of followers scored the least. The highest ranked factor was the dentist's qualifications followed by before and after pictures of treated cases then positive comments or recommendations from the followers. These results reflect the awareness of the society about the importance of professional qualifications rather than commercial accounts that can manipulate the actual number of followers to give an unrealistic impression of popularity. About fifty eight percent of the respondents reported that they do search the social media if they ever faced any dental issue, and $68.6 \%$ of them reported that they usually find the information they searched for. And again females were the majority with positive responses $(62 \%)$ in comparison to males $(46.5 \%)$. These results validate the effectiveness of social media in relation to dentistry. Only $19.4 \%$ of participants reported that they shared their personal experience on social media, whether it was related to treatment modality or with a specific dentist. Encouragement and motivation to the society to share their experience and opinion helps in spreading the knowledge. About half of the participants $(42.5 \%)$ reported that they don't follow any dental accounts on social media, and $40.3 \%$ are following one to five dental accounts. These results have enlightened the need to encourage the dental professionals to invest more time and effort in making their social media accounts more attractive and interactive. The need to have a specialized dental websites is represented when most of the participants $(48.4 \%)$ believed that it is the most effective method to gain information related to dentistry. This result showed that people does not depend solely on social media as a source of information, and we see the effectiveness of professional dental websites since the interest between social media platform is changing rapidly in short periods of time and new platforms could be introduced in the coming years. Professional websites are timeless and more trust worthy. This result was in contrast to a previous study conducted in North America in 2011, where they stated that "social media, with its ability to target individual patients with information tailored to their unique interests, may eventually surpass the standard website (which is far more static) as the preferred method of marketing. This seems likely in much the same way that practice websites were not as powerful or prevalent 10 years ago as they are today". ${ }^{18}$

\section{Conclusion}

Social media is bringing a new dimension to the health care area by offering a medium to be used by patients, and health care professionals. These networks are bringing health information to many more people than ever before. And they are broadening the access to those who may not easily retrieve health information via traditional methods, such as younger people, minorities, and lower socioeconomic groups. Clearly, more research is needed on the potential use and outcomes of social media applications in dental knowledge. Nevertheless, the use of social media is rapidly growing, and there may be good opportunities for dental knowledge awareness.

\section{Funding}

None.

\section{Acknowledgments}

The author would like to express the deepest appreciation to Professor Nahid Ashri, Department of Periodontics and Community Dentistry, College of Dentistry, King Saud University for her support in executing this study and her advice in writing the manuscript.

\section{Conflicts of interest}

The author has no conflicts of interest to declare.

\section{References}

1. Von Muhlen M, Ohno-Machado L. Reviewing social media use by clinicians. J Am Med Inform Assoc. 2012;19(5):777-781

2. Chretien KC, Kind T. Social Media and Clinical Care. Ethical, Professional, and Social Implications. Circulation. 2013;127(13): 14131421

3. Penetration of leading social networks in Saudi Arabia as of 4th quarter 2016. Statista. 2017.

4. Ventola CL . Social Media and Health Care Professionals: Benefits, Risks, and Best Practices. 2014;PT 39(7):491-520.

5. Michele P Hamm, Annabritt Chisholm, Jocelyn Shulhan, et al. Social media use among patients and caregivers: a scoping review. BMJ Open. 2017;3(5):1-10

6. Househ ME, Borycki A, Kushniruk. Empowering patients through social media: The benefits and challenges. Health Informatics J. 2014;20(1):50 58 .

7. Kaplan AM, Haenlein M. Users of the world, unite! The challenges and opportunities of Social Media. Business Horizons. 2017;53(1):59-68

8. Eckler PG, Worsowicz, Rayburn JW. Social Media and Health Care: An Overview. PM R. 2010;2(11):1046-1050.

9. Lenhart A, Kristen Purcell, Smith A. Social media \& mobile Internet use among teens and young adults, Pew Research Center, USA. 2010.

10. Korda H, Itani Z. Harnessing Social Media for Health Promotion and Behavior Change. Health Promot Pract. 2013;14(1):15-23. 
11. Timimi FK. Medicine, morality and health care social media. $B M C$ Medicine. 2012;10(1): 83.

12. Fox S. Health topics. Pew Research Center, USA. 2011.

13. Fox S, Duggan M. Health online 2013, Pew Research Center, USA. 2013

14. Duggan M, Brenner J. The demographics of social media users-2012. Pew Research Center, USA, 2013.

15. Currell R, Urquhart C, Wainwright $\mathrm{P}$, et al. Telemedicine versus face to face patient care: effects on professional practice and health care outcomes. Cochrane Database Syst Rev. 2000;(2):CD002098.

16. Duggan M. The demographics of social media users. 2015.

17. Susannah Fox. Maeve Duggan Health Online 2013, Pew Research Center, USA. 2013.

18. Wheeler CK, Said H, Prucz R, et al. Social media in plastic surgery practices: emerging trends in North America. Aesthet Surg $J$ 2011;31(4):435-441.

19. Keckley P. Survey of Health Care Consumers in the United States: Key Findings, Strategic Implications. Deloitte Center for Health Solutions, USA. 2011; p. 1-30.

20. The Health Communicator's Social Media Toolkit, Centers for Disease Control and Prevention. 2011.
21. Sarasohn-Kahn J .The wisdom of patients: health care meets online social media. California Health care Foundation, California, USA, 2008.

22. Hawn C. Take two aspirin and tweet me in the morning: How Twitter, Facebook, and other social media are reshaping health care. Health Aff (Millwood). 2008;28(2):361-368.

23. Crocco AG, Villasis-Keever M, Jadad AR. Two wrongs don't make a right: harm aggravated by inaccurate information on the Internet. Pediatrics. 2002;109(3):522-523.

24. Chou WY, Prestin A, Lyons C, et al. Web 2.0 for Health Promotion: Reviewing the Current Evidence. Am J Public Health. 2013;103(1):e9e18.

25. Crocco AG, Villasis-Keever M, Jadad AR . Analysis of cases of harm associated with use of health information on the internet. JAMA. 2002;287(21):2869-2871.

26. Greysen SR, Kind T, Chretien KC. Online Professionalism and the Mirror of Social Media. J Gen Intern Med. 2010;25(11):1227-1229.

27. Kemp S. Digital in 2017: Global Overview. 2017.

28. World Internet Users and 2017 Population Stats, Miniwatts Marketing Group. 2017.

29. Saudi Arabia Social Media Statistics 2016, Gmi blogger, UAE. 2016. 